\title{
Evidence for Thalamocortical Circuit Abnormalities and Associated Cognitive Dysfunctions in Underweight Individuals with Anorexia Nervosa
}

\author{
Dominik Biezonski*, $^{*,}$ Jiook Cha', Joanna Steinglass ${ }^{1,2}$ and Jonathan Posner ${ }^{1,2}$ \\ 'Department of Psychiatry, Columbia University College of Physicians and Surgeons, New York State Psychiatric Institute, New York, NY, USA
}

\begin{abstract}
Anorexia nervosa (AN) is characterized by extremely low body weight resulting from pathological food restriction, and carries a mortality rate among the highest of any psychiatric illness. AN, particularly during the acute, underweight state of the illness, has been associated with abnormalities across a range of brain regions, including the frontal cortex and basal ganglia. Few studies of AN have investigated the thalamus, a key mediator of information flow through frontal-basal ganglia circuit loops. We examined both thalamic surface morphology using anatomical MRI and thalamo-frontal functional connectivity using resting-state functional MRI. Individuals with $A N(n=28)$ showed localized inward deformations of the thalamus relative to healthy controls $(\mathrm{HC}, n=22)$, and abnormal functional connectivity between the thalamus and the dorsolateral and anterior prefrontal cortices. Alterations in thalamo-frontal connectivity were associated with deficits in performance on tasks probing cognitive control (Stroop task) and working memory (Letter-Number Sequencing (LNS) task). Our findings suggest that abnormalities in thalamo-frontal circuits may have a role in mediating aspects of cognitive dysfunction in underweight individuals with AN.
\end{abstract}

Neuropsychopharmacology (2016) 4I, I560-I568; doi:I0. I038/npp.20I5.3I4; published online 4 November 2015

\section{INTRODUCTION}

Anorexia nervosa (AN) is a serious disorder characterized by aberrant eating behavior and inappropriately low body weight, and is associated with a mortality rate among the highest of any psychiatric illness (Swanson et al, 2011). Neuroimaging research has demonstrated metabolic, morphological, and functional connectivity abnormalities among patients with $\mathrm{AN}$ in frontal, temporal, and visual cortical regions, as well as within subcortical structures including the basal ganglia (Amianto et al, 2013; Cowdrey et al, 2014; Delvenne et al, 1995; Husain et al, 1992; Matsumoto et al, 2006; Takano et al, 2001; Yau et al, 2013). Relatively few studies, however, have investigated the thalamus, a subcortical region that, in part, relays output from the basal ganglia to the cortex through parallel corticostriato-thalamo-cortical (CSTC) loops (Alexander and Crutcher, 1990; Haber and Calzavara, 2009; Parnaudeau et al, 2013).

Studies of individuals with AN have previously documented signs of thalamic dysfunction, particularly during the acute, underweight phase of the illness. Amianto et al (2013)

*Correspondence: Dr D Biezonski, New York State Psychiatric Institute, Columbia University, I05I Riverside Dr, Unit 74, Room 2420, New York, NY 10032, USA, Tel: + 646774 5879, Fax: + I 314 27I 2446, E-mail: biezons@nyspi.columbia.edu

${ }^{2}$ Shared senior authors.

Received 24 June 2015; revised 29 September 2015; accepted 29 September 2015; accepted article preview online 14 October 2015 used anatomical MRI and voxel-based morphometry and reported reduced gray matter in a cluster overlapping the right thalamus. Moreover, single-photon emission computed tomography has shown hyperperfusion in the thalamus in AN, indicative of hypermetabolism (Takano et al, 2001). One cross-sectional diffusion tensor imaging study of low weight and weight-restored individuals with AN reported altered white-matter integrity in the left and right posterior thalamic radii (Frieling et al, 2012). These data suggest that patients with AN may exhibit thalamic abnormalities at the level of morphology as well as thalamocortical connectivity.

Emerging evidence suggests that neural connections between the thalamus and the frontal cortex (ie, thalamofrontal circuits) may subserve executive functions including set-shifting, working memory, and cognitive control (Hughes et al, 2012; Marenco et al, 2012; Mitelman et al, 2006; Parnaudeau et al, 2013). These executive functions may be disrupted in patients with AN (Bodell et al, 2014; Park et al, 2014; Steinglass et al, 2006; Talbot et al, 2014). However, the relationship between alterations in thalamo-frontal circuits and cognitive dysfunction in AN has not been fully explored.

The primary aim of this study was to investigate whether the thalamus shows abnormalities in morphology or functional connectivity among individuals with AN relative to healthy controls (HC). Using anatomical MRI scans, we performed surface analysis of the thalamic structure. Surface analysis examines discrete areas of deformation computed from group differences in the shape of a defined brain region, in this case the thalamus. Since different regions of 
Table I Participant Demographics and Clinical Characteristics

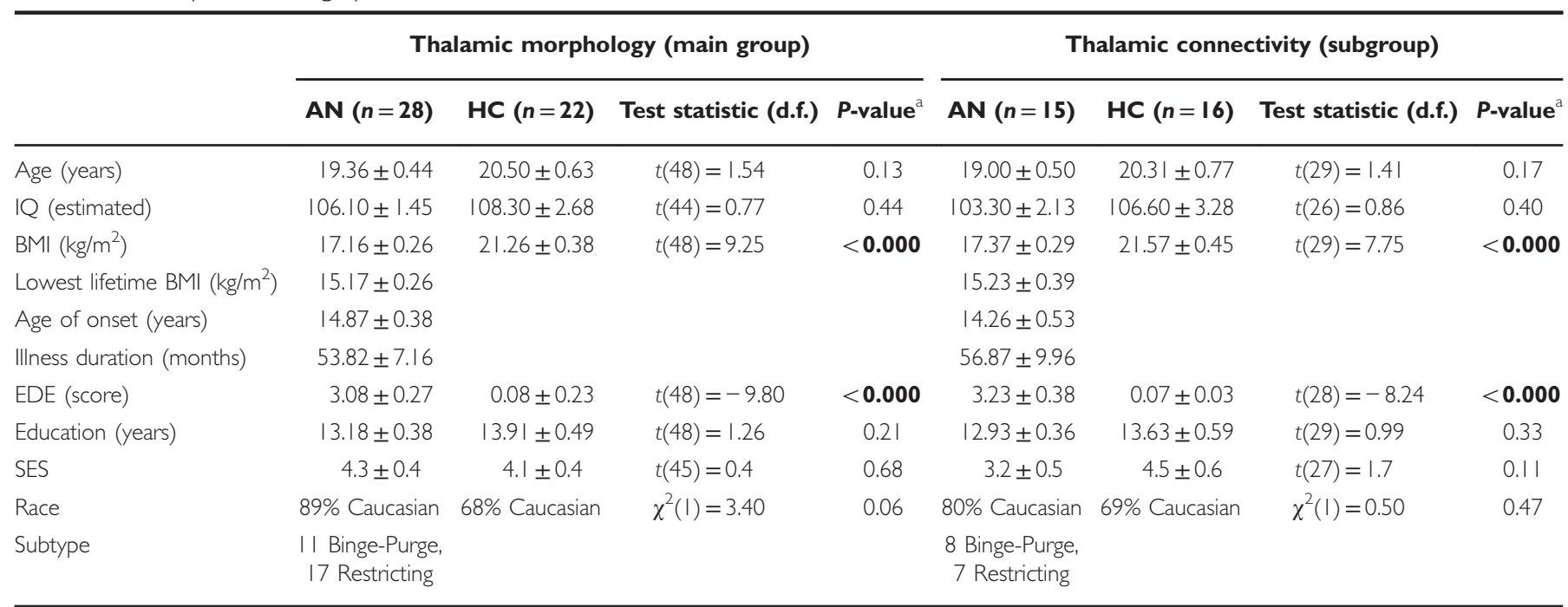

Abbreviations: AN, anorexia nervosa; BMI, body mass index; EDE, Eating Disorder Examination Interview; HC, healthy control; IQ, full-scale IQ estimated by the Wechsler Test of Adult Reading (WTAR); SES, socioeconomic status. Data are presented as mean \pm SEM (see Supplementary Table SI for scoring details).

${ }^{a}$ Two-tailed in case of $t$-tests.

$P$-values in bold denote statistical significance $\leqslant 0.05$

the thalamus project to discrete regions of the cortex (Johansen-Berg et al, 2005), this analysis can reveal potential abnormalities in specific thalamocortical circuits and their related cognitive functions. Resting-state functional MRI scans were used to assess group differences in functional connectivity, a measure of the coherence of fMRI signal across brain regions, between the thalamus and the frontal cortex. We hypothesized that relative to $\mathrm{HC}$, individuals with AN would show localized alterations in thalamic morphology, as well as abnormal functional connectivity within thalamo-frontal circuitry. We also explored whether thalamic morphology and functional connectivity correlated with performance on cognitive tasks that probe executive functions.

\section{MATERIALS AND METHODS}

The Institutional Review Board of the New York State Psychiatric Institute approved the study procedures. Adult participants provided written informed consent. Adolescent participants provided informed assent, and a legal guardian provided written informed consent.

\section{Participants}

Study subjects were included if they had participated in a neuroimaging study of AN described elsewhere (Decker et al, 2014). Participants were females with AN, aged 16-25 years, receiving in-patient treatment through the Eating Disorders Research Clinic at the New York State Psychiatric Institute (NYSPI)/Columbia University Medical Center, and groupmatched HC (Table 1). Individuals with AN participated in this study within their first week of hospital admission, and were medically stable as determined by their clinical team. Eligible patients met DSM-5 (APA, 2013) criteria for AN, both restricting (AN-R) and binge-purging (AN-BP) subtypes. Individuals were excluded if they had an estimated
IQ of $<80$; a history of a neurological, bipolar, or psychotic disorder; substance abuse in the last 6 months; were currently taking psychotropic medication; had contraindications to MRI; or if they were pregnant. Anxiety or depressive disorders, which commonly co-occur with AN (Godart et al, 2007), were not exclusionary when $A N$ was the primary diagnosis. HC were group matched to the participants with AN by age, sex, IQ, and ethnicity, and had a body mass index $(\mathrm{BMI})$ in the normal range $\left(18-25 \mathrm{~kg} / \mathrm{m}^{2}\right)$. Additional exclusion criteria for $\mathrm{HC}$ were any current or past psychiatric illness, significant medical illness, or history of psychotropic medication.

\section{Neuroimaging and Cognitive Testing: Overview}

Participants (AN, $n=28$; HC, $n=22$ ) were scanned with anatomical MRI and a subgroup of participants (AN, $n=15$; HC, $n=16$ ) also underwent resting-state functional MRI. All participants underwent cognitive testing as part of a neuropsychological battery within 1 week of MRI scanning.

\section{Anatomical MRI Pulse Sequences}

Images were acquired on a 1.5-T Philips Intera scanner with an 8-channel head coil. Acquisition of T1-weighted sagittal localizing images was followed by a $3 \mathrm{D}$ spoiled gradient recall (SPGR) image using the following parameters: $\mathrm{TR}=$ $25 \mathrm{~s}, \mathrm{TE}=3.7 \mathrm{~ms}$, flip angle $=30^{\circ}, \mathrm{FOV}=256 \mathrm{~mm}, 256 \times 204$ matrix, 128 slices, voxel size $1 \times 1 \times 1 \mathrm{~mm}$.

\section{Resting-State Functional MRI Pulse Sequences}

Two 5-min resting-state scans were obtained using the following parameters: $\mathrm{TR}=2000 \mathrm{~ms}$, $\mathrm{TE}=40 \mathrm{~ms}$, flip angle $=$ $77^{\circ}, 33$ slices, voxel size $=3 \times 3 \times 4 \mathrm{~mm}, 150$ volumes. During scan acquisition, participants were instructed to stay still with their eyes open, and to let their minds wander freely. 


\section{Processing of Surface Morphology using Vertex Analysis}

To perform semi-automated segmentation and surface vertex analysis of subcortical structures in each participant, we used the FIRST pipeline through FSL (v5.0, fsl.fmrib.ox.ac.uk/fsl/ fslwiki) (Patenaude et al, 2011). This method uses the 'active appearance model' within a Bayesian framework to segment 15 subcortical structures. The structures are defined a priori by 336 manually traced 'training' images, after which vertex analysis is used to compute local differences in structural morphology across groups. Briefly, high-resolution T1weighted anatomical images for each subject, as well as the training images, were subjected to linear affine registration followed by non-linear registration to the Montreal Neurological Institute (MNI) 152 standard template using FSL FLIRT and FNIRT (Jenkinson et al, 2012; Patenaude et al, 2011). Manually delineated training image structures were then modeled by fitting a deformable mesh consisting of triangle vertices connected by edges with known threedimensional coordinates. Subject-specific subcortical regions were then modeled by fitting a triangle/vertex mesh to the overlapping locations of the corresponding vertices derived from the training data. The subcortical regions of interest (ie, left and right thalamus) modeled for every participant were then concatenated in a single four-dimensional file, and vertex analysis was performed using rigid alignment in MNI152 standard space to estimate local variation in thalamic vertex position (and thus localized thalamic deformations) between groups. Brain size differences were controlled for by normalizing and registering each participant's brain scan to MNI152 standard space using the FLIRT pipeline within FSL (Jenkinson et al, 2002; Jenkinson and Smith, 2001). For subsequent correlation analyses, we used SPM8 (Statistical and Parametric Mapping, v8, www.fil.ion. ucl.ac.uk/spm/software/spm8) to extract the average thalamic deformation value for each participant.

\section{Processing of Resting-State Functional Connectivity}

Computation of resting-state functional MRI-based connectivity maps was performed using the functional connectivity toolbox (Conn, v14, www.nitrc.org/projects/conn) as detailed in Whitfield-Gabrieli and Nieto-Castanon (2012). Briefly, anatomical and fMRI images for each participant were spatially pre-processed using Artifact Detection Tools (Art, www.nitrc.org/projects/artifact_detect) through SPM8 to perform slice-timing correction, realignment, co-registration, spatial smoothing, and CompCor-based noise removal. Estimated temporal confounds including head motion and background blood oxygen level-dependent (BOLD) signal intrinsic to white matter and cerebral spinal fluid were used as covariates in the BOLD time series to regress out non-neural sources of BOLD signal. Finally, head motion parameters $\mathrm{FD}_{\text {PEAK, }} \mathrm{FD}_{\text {MEAN }}$, DVARS $\mathrm{PEAK}_{\text {, and }}$ DVARS MEAN (see Supplementary Methods for description, and Supplementary Table S2 for group comparisons) were estimated and extracted using SPM 8 for use as covariates in all subsequent analyses involving functional connectivity results.

Seven bilateral thalamic seed masks, each corresponding to a thalamic region with preferential connections to a specific cortical region or lobe (Johansen-Berg et al, 2005), were thresholded at $25 \%$ connection probability, binarized, and extracted from the Oxford thalamic connectivity atlas (OTCA) included with FSL (Supplementary Figure S1). We used bilateral masks as (a) the thalamus has been shown to be bilaterally engaged during the performance of tasks probing the same cognitive domains as were examined in our study (Forsyth et al, 2014; Hendrick et al, 2010; Moore et al, 2013; Wagner et al, 2013) and (b) the thalamus in the AN group showed evidence of bilateral morphometric deformation (see Results). For each subject, functional connectivity maps were estimated using voxel-wise seed-to-voxel bivariate correlations between the BOLD time series of each thalamic seed and the whole brain. To improve data normality for second-level analysis using the General Linear Model, a Fisher-z transform was applied to the resulting connectivity data. We limited our analyses to thalamic connectivity with the frontal lobes (ie, Brodmann areas (BA) 8-10, 45-47) because of our goal of examining the contribution of altered thalamo-frontal circuitry to executive dysfunction in AN (Hughes et al, 2012; Marenco et al, 2012; Mitelman et al, 2006; Parnaudeau et al, 2013).

We used SPM8 to extract the average connection strength for each thalamo-frontal cluster in which significant differences in connectivity were detected between patients with AN and HC. Brain coordinates for each cortical cluster were used to find the nearest local maxima within each cluster. The average connectivity values within a 6-mm radius of this peak coordinate were then extracted as eigenvalues for each cluster and compared with outcomes of cognitive performance, as well as various control measures, for each subject as described in statistical analyses.

\section{Cognitive Measures}

The neuropsychological battery included the Stroop task to assess cognitive control (Golden, 1978), the LNS task to measure working memory (WAIS-III; Wechsler, 1997), and the Trail-Making Test (TMT) to assess processing speed and visual attention (Bowie and Harvey, 2006). The administration and scoring of these tasks are included in Supplementary Methods.

\section{Hypothesis Testing}

Vertex analysis in FSL FIRST was used to compute morphological thalamic differences between participants with $\mathrm{AN}$ and $\mathrm{HC}$ using the General Linear Model through the randomize function in FSL. Thalamic morphology was used as a dependent variable, while group designation was used as the independent variable. We used the HC-AN contrast to measure the group difference in inward thalamic deformation, and the $\mathrm{AN}-\mathrm{HC}$ contrast to measure the group difference in outward deformation, both at a family-wise error (FWE)-corrected $P \leqslant 0.05$ using threshold-free cluster enhancement. This analysis was then repeated while controlling for age and IQ.

For each thalamic seed, group differences in whole brain seed-to-voxel resting-state functional connectivity were independently estimated using the General Linear Model through SPM8. Resting-state connectivity maps for each of the seven thalamic seeds (Supplementary Figure S1) were used as the dependent variable, while group designation was 

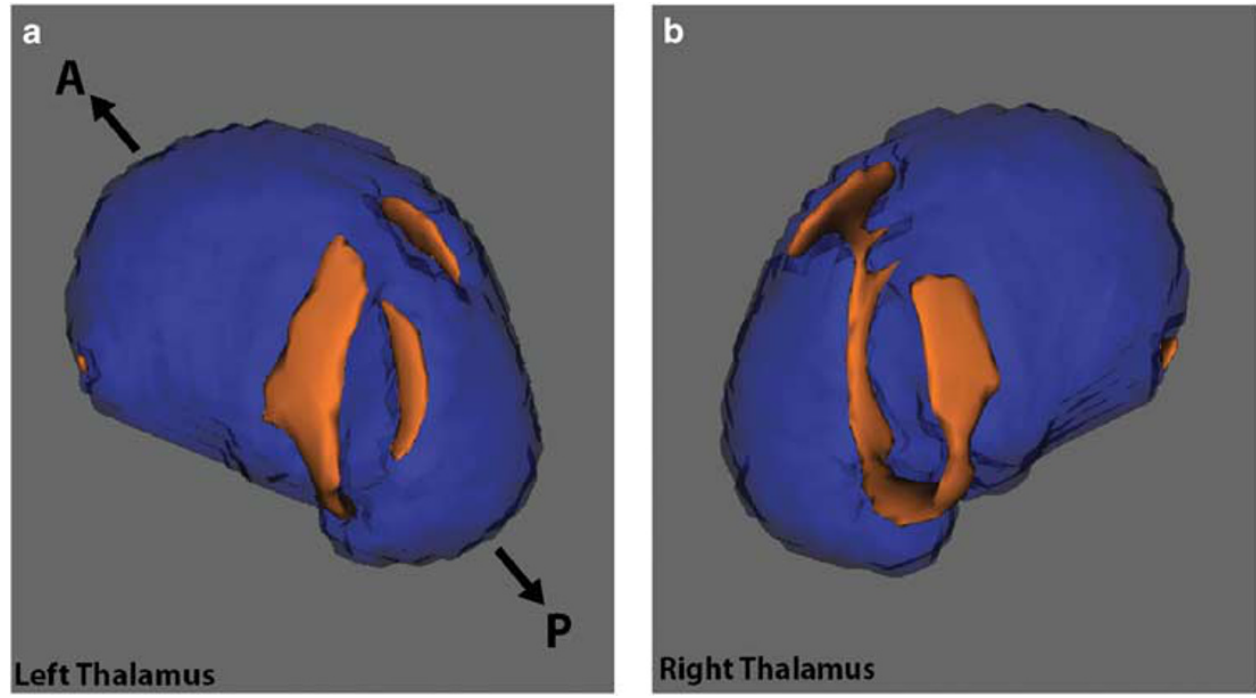

Figure I Thalamic morphology. Compared with healthy controls, patients with anorexia nervosa showed inward surface deformations (orange) spanning the mid dorsal-ventral aspects of the left (a) and right (b) thalamus (blue) $\left(P_{F W E}<0.05\right)$. Arrows indicate anterior-posterior ('A', ' $P$ ') orientation of the thalamic structure.

used as the independent variable. The false-positive rate was controlled by both a voxel-level threshold of $P=0.005$ and a cluster extent threshold using false discovery rate (FDR) correction. $P_{\mathrm{FDR}} \leqslant 0.05$ was considered as statistically significant. Bonferroni correction was used to control for multiple comparisons stemming from independently examining each of the seven thalamic seeds. These analyses were then repeated while controlling for age, IQ, and head motion parameters.

\section{Exploratory Analyses}

Two sample $t$-tests were used to assess group differences in performance on the Stroop, TMT, and LNS between the individuals with $\mathrm{AN}$ and $\mathrm{HC}$, who were included in the resting-state functional connectivity analysis. This analysis was then repeated using the general linear model to control for age and IQ.

Partial correlations were used to compute the association between thalamo-frontal functional connectivity strength, and performance on all three cognitive tasks across all subjects while controlling for group (AN or $\mathrm{HC}$ ), age, IQ, and head motion parameters. Partial correlations were also used to compute the association between (a) thalamic morphology and thalamo-frontal functional connectivity strength and (b) between thalamic morphology and performance on cognitive tasks. For these exploratory analyses, a $P \leqslant 0.05$ was considered as statistically significant. Potential effects of outliers were controlled for using the bias-corrected and accelerated method on bootstrapped samples $(n=1000)$.

\section{Sensitivity Analyses}

We performed several sensitivity analyses to examine the potential impact of $\mathrm{AN}$-associated malnutrition, illness severity, and comorbid diagnoses on our findings, detailed in Supplementary Information. More specifically, we examined the extent to which AN subtype, current BMI, lowest lifetime BMI, illness duration, comorbid diagnoses, and average low-frequency fluctuations (ALFF) in BOLD signal may have influenced our study outcomes.

\section{RESULTS}

\section{Study Participants}

Clinical characteristics are described in Table 1. Groups differed significantly in BMI $(P<0.001)$ and EDE scores $(P<0.001)$, but did not differ in age, IQ, years of education, socioeconomic status (SES), or race/ethnicity. The subgroup of participants who were included in resting-state functional MRI did not differ significantly from the full sample (see Supplementary Table S1). Among the individuals with AN, eight patients met criteria for a comorbid psychiatric illness (three with specific phobia, four with major depressive disorder (MDD), and one with post-traumatic stress disorder). The same AN participants with comorbid diagnoses were also part of the subgroup, excluding one individual with MDD.

\section{Hypothesis Testing}

Thalamic morphology. Compared with HC, patients with AN exhibited significant inward deformations in several regions of the left and right thalamus, spanning the central dorsal-ventral aspects of the thalamus both medially and laterally $\left(P_{\mathrm{FWE}}<0.05\right.$, Figure 1$)$. The group difference remained significant after controlling for the effects of age and IQ $\left(P_{\mathrm{FWE}}<0.05\right)$.

Thalamo-frontal functional connectivity. Relative to HC, individuals with AN showed greater connectivity between the central-medial thalamus (OTCA thalamic seed 5, 'Thal5', Supplementary Figure S1) and the bilateral dorsolateral prefrontal cortex (DLPFC, BA9/BA46; Thal5-DLPFC(L) and Thal5-DLPFC(R), Figure 2a and b), and lower functional 

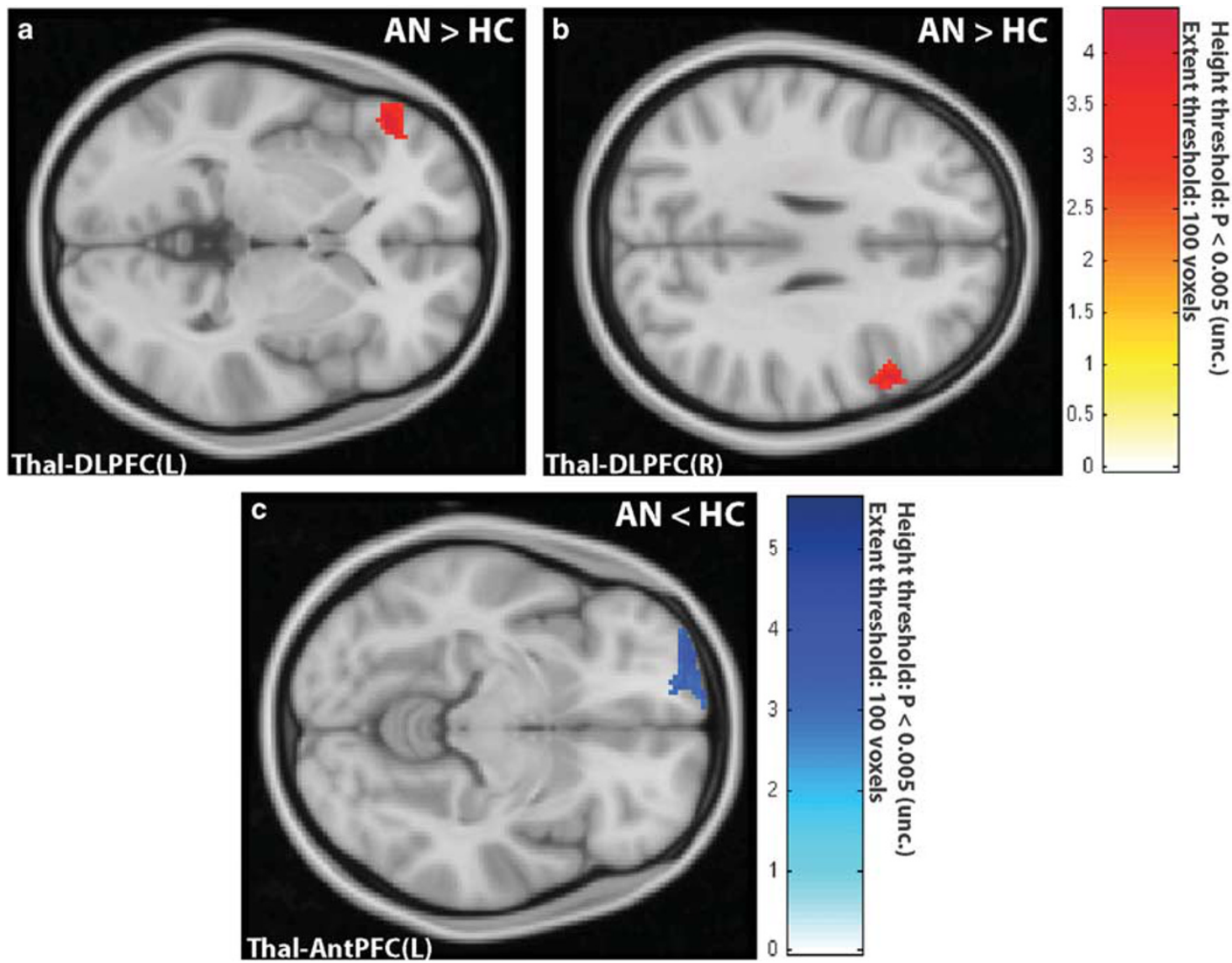

Figure 2 Thalamo-frontal functional connectivity. Relative to healthy controls ( $\mathrm{HC})$, individuals with anorexia nervosa (AN) exhibited abnormally high functional connectivity between the thalamus and the left dorsolateral prefrontal cortex (Thal-DLPFC(L)) (a) as well as right DLPFC (Thal-DLPFC(R)) (b) $\left(P_{\text {FDR }}<0.05\right)$. The yellow-red bar scale indicates increasingly higher group connectivity differences in the positive direction (ie, AN $\left.>H C\right)$. (c) Conversely, relative to $\mathrm{HC}$, individuals with $\mathrm{AN}$ exhibited abnormally low functional connectivity between the thalamus and the left anterior prefrontal cortex (ThalAntPFC $(L))\left(P_{\mathrm{FDR}}<0.05\right)$. The white-blue bar scale indicates increasingly higher group connectivity differences the negative direction (ie, $\mathrm{AN}<\mathrm{HC}$ ).

connectivity between the anterior thalamus (OTCA thalamic seed 4, 'Thal4') and the left anterior prefrontal cortex (AntPFC, BA10; Thal4-AntPFC(L), Figure 2c). The group differences remained significant after controlling for the effects of age, IQ, and head motion parameters $\left(P_{\mathrm{FDR}}<0.05\right)$, as well as Bonferroni correction for multiple seed comparisons (threshold $P=0.0071$; cluster $P$ 's $s_{\mathrm{unc}}<0.001$ ).

\section{Exploratory Analyses}

Cognitive performance. Compared with $\mathrm{HC}$, patients with AN showed significant impairment in performance on the Stroop task $(t(28)=1.78, P=0.04)$ and the LNS task $(t(28)=$ $1.71, P=0.05)$, but not the TMT $(P=0.28)$ (Table 2); these results remained significant after controlling for age and IQ $\left(P^{\prime} s \leqslant 0.05\right)$.

MRI measures and cognitive performance. Partial correlations controlling for age, IQ, group (AN or $\mathrm{HC}$ ), and head motion revealed a significant negative correlation between performance on the Stroop and both the Thal5-DLPFC(L) and Thal5-DLPFC(R) connectivity strength (Stroop vs Thal5-DLPFC(L): $r(21)=-0.45, P=0.02$; Stroop $v s$ Thal5DLPFC(R): $r(21)=-0.38, P=0.04$; Figure $3 \mathrm{a}$ and $\mathrm{b}$ ). Lower scores on the Stroop reflect higher interference and greater impairment. Group-wise bivariate correlations revealed a strong trend toward negative correlation between Stroop performance and Thal5-DLPFC(L) as well as Thal5-DLPFC (R) connectivity strength in AN (Stroop $v s$ Thal5-DLPFC(L): $r(14)=-0.44, \quad P=0.06 ; \quad$ Stroop $\quad v s \quad$ Thal5-DLPFC(R): $r(14)=-0.43, \quad P=0.06$ ) but not in HC (Stroop vs Thal5-DLPFC(L): $\quad P=0.10 ; \quad$ Stroop $\quad v s$ Thal5-DLPFC(R): $P=0.26)$.

Partial correlations controlling for age, IQ, group (AN or $\mathrm{HC}$ ), and head motion revealed a significant positive correlation between performance on the LNS task and Thal4-AntPFC(L) connectivity $\quad(r(21)=0.58, \quad P=0.002$; Figure $3 \mathrm{c}$ ), where higher LNS scores reflect better performance. Group-wise bivariate correlations revealed a significant positive correlation between LNS performance and Thal4-AntPFC(L) connectivity strength in $\mathrm{AN}(r(15)=0.45$, $P=0.05)$ but not in HC $(P=0.14)$.

No significant association was found between performance on the TMT and thalamo-frontal connectivity $(P$ 's $>0.10)$. Thalamic morphology did not correlate with measures of thalamo-frontal connectivity nor performance on any of the cognitive measures $\left(P^{\prime} \mathrm{s}>0.14\right)$. 
Table 2 Group Comparisons on Cognitive Task Performance

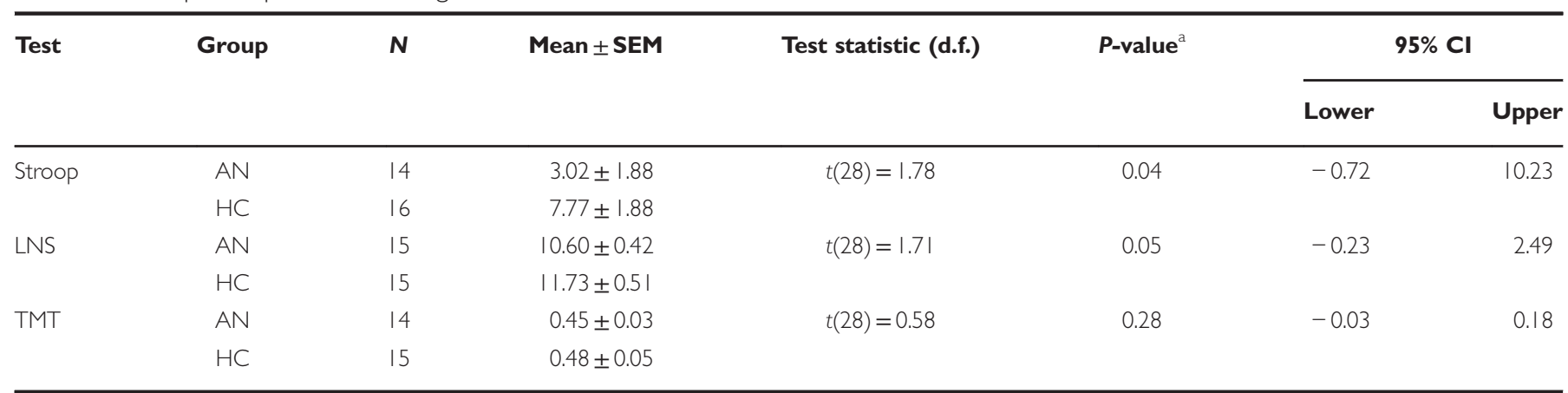

Abbreviations: AN, anorexia nervosa; Cl, confidence interval; HC, healthy control; LNS, Letter-Number Sequencing task; TMT, Trail-Making Test.

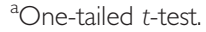
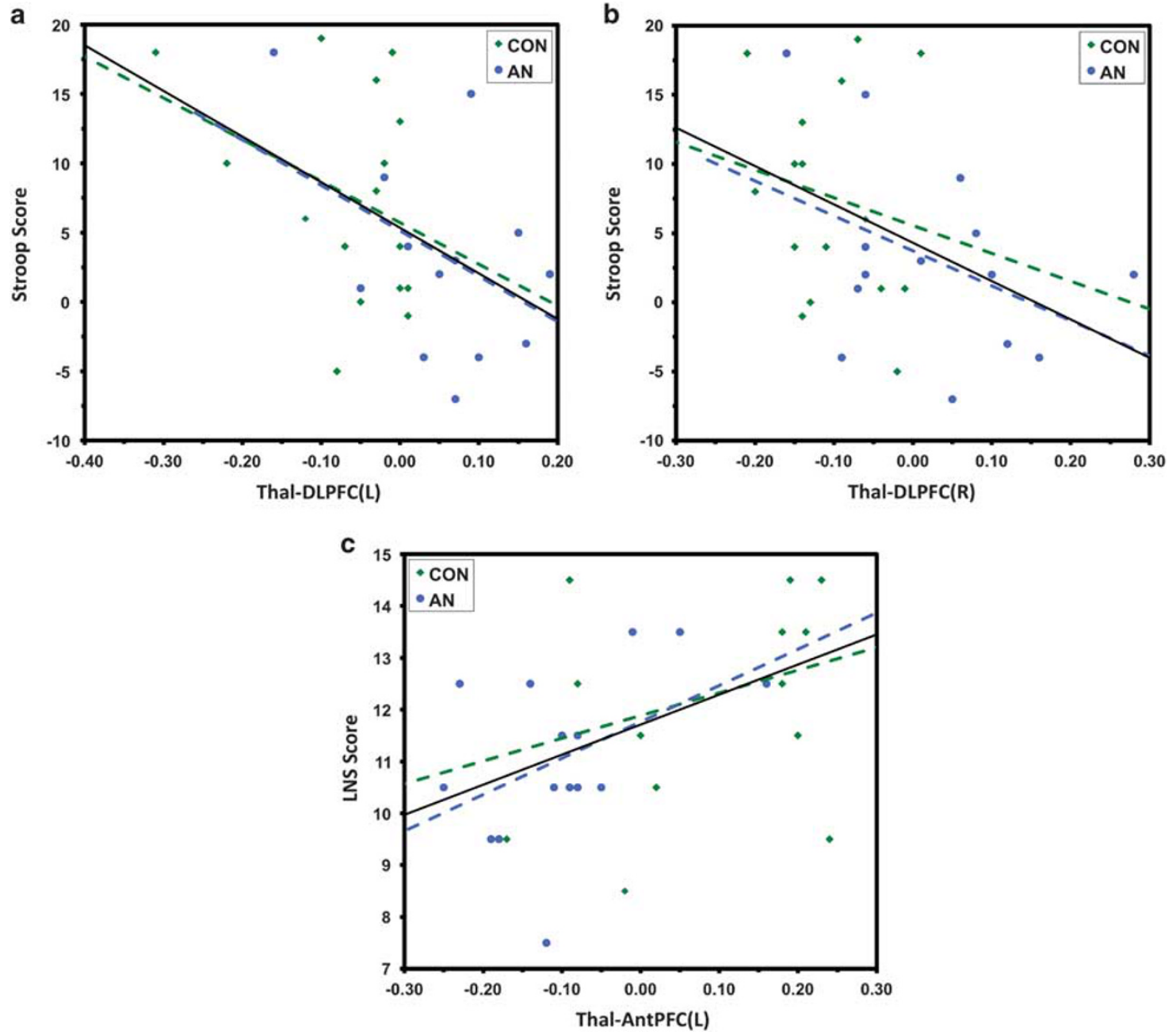

Figure 3 Thalamo-frontal functional connectivity and cognitive performance. Partial correlations controlling for age, IQ, group (AN or HC), and head motion revealed a significant negative correlation between performance on the Stroop task and connection strength between the thalamus and both the left dorsolateral prefrontal cortex (Thal-DLPFC $(L))(a), r(2 I)=-0.45, P=0.02$ ) and right DLPFC (Thal-DLPFC(R)) $(b), r(2 I)=-0.38, P=0.04), w h e r e$ lower scores on the Stroop reflect higher interference and greater impairment. These analyses also revealed a significant positive correlation between performance on the Letter-Number Sequencing (LNS) task and thalamic-anterior prefrontal cortex (Thal-AntPFC(L)) connectivity $(c), r(2 \mathrm{I})=0.56, P=0.002)$, where higher LNS scores reflect better performance. Colored perforated lines are fitted to group-wise data, while the solid black line is fitted to data across both groups. 


\section{Sensitivity Analyses}

Results from our sensitivity analyses are detailed in Supplementary Information.

\section{DISCUSSION}

Using anatomical and resting-state functional MRI, we examined surface morphology and functional connectivity of the thalamus in patients with AN in comparison with HC. Individuals with AN in the acute, underweight phase of the illness exhibited altered thalamic surface morphology, as well as abnormal functional connectivity between the thalamus, the DLPFC, and the AntPFC. The alterations in thalamofrontal connectivity were associated with impairments in performance on tasks probing working memory and cognitive control, but not visuospatial processing speed and attention.

Compared with HC, individuals with AN showed localized inward deformations in bilateral thalamus. These deformations may result from a number of factors, including cellular loss, atrophy of neuronal dendritic arbors, demyelination, reduction in somatic size, or loss of afferent input (Sowell et al, 2004). In rodents, it has been shown that increased neuronal excitability is associated with a decrease in dendritic complexity (Cazorla et al, 2012). One previous study has shown hypermetabolism in the thalamus in AN (Takano et al, 2001), which may reflect higher intrinsic neural activity, and potentially explain the thalamic deformation seen in our study as resulting from dendritic atrophy. If thalamic metabolism normalizes following weight restoration, as suggested by two prior studies of AN (Delvenne et al, 1996; Frank et al, 2007), then thalamic deformations in AN may also normalize with successful treatment. Alternatively, these deformations may represent loss in cellular number, myelination, or synaptic input into the thalamus, the analysis of which would require post-mortem tissue.

Evidence from both animal and human studies suggests that the DLPFC, AntPFC, and anterior cingulate gyrus act in concert during various executive functions including working memory, set-shifting and behavioral flexibility, attention, and cognitive control (Botvinick et al, 2001; MacDonald et al, 2000; Milham et al, 2003; Niendam et al, 2012; Parnaudeau et al, 2013; Van Snellenberg et al, 2014). Modulation of these cognitive domains is achieved by parallel processing through CSTC loops, the final output of which involves thalamic interactions with frontal regions (Ferguson and Gao, 2014; Giguere and Goldman-Rakic, 1988; Graybiel, 2005; Haber and Calzavara, 2009; Parnaudeau et al, 2013). Our study found that when compared with HC, individuals with AN exhibited altered thalamo-frontal functional connectivity that was associated with deficits in performance on tasks probing cognitive control and working memory. This suggests that altered thalamo-frontal connectivity may have a role in mediating aspects of cognitive dysfunction in this disorder. In this regard, it is noteworthy that in a recent study in rodents, pharmacogenetic disruption of thalamo-frontal interactions yielded deficits in similar cognitive domains (Parnaudeau et al, 2013).

We did not find associations between localized thalamic deformations and either thalamo-frontal functional connectivity or executive function performance. This suggests that either thalamic morphometry may be independent of alterations in thalamo-frontal functional connectivity, or the included measures lack the sensitivity needed to detect such an association. Little is known about the relationship between structural changes in the thalamus and their effect on thalamic function, and future investigations are needed to clarify how thalamic morphology and function interact to ultimately influence cognition and behavior.

In this study, we examined the acute, underweight state of AN, when it is difficult to discern the impact of malnutrition alone from disease-specific processes. To address this issue, we performed two sensitivity analyses in which (a) we examined the potential impact of malnutrition on our resting-state connectivity findings by assessing group differences in the ALFF in blood-oxygen-level dependent (BOLD) signal, a proxy measure of glucose metabolism in the brain (Nugent et al, 2015; Tomasi et al, 2013), within the thalamus, AntPFC, and DLPFC and (b) we assessed the extent to which current BMI, one index of malnutrition, correlated with our main study findings in the AN group. We found no group differences in ALFF within any of the examined thalamofrontal regions, suggesting that the AN group did not exhibit alterations in BOLD signal that may have confounded the results of our resting-state connectivity analyses. In addition, current BMI in the AN group did not correlate with the extent of deformation in the left or right thalamus, or Thal4AntPFC connectivity. However, this measure did significantly correlate with Thal5-DPLFC functional connectivity, bilaterally. Given this finding, it is conceivable that connectivity dynamics within certain thalamo-frontal circuits are more sensitive to the effects of malnutrition than others; by implication, these circuits may also be more amenable to normalization in response to weight restoration irrespective of AN diagnosis. Nonetheless, future work will be needed to fully establish the role of thalamic dysfunction in the cognitive and behavioral disturbances that are central to AN.

The present study examined morphological and functional connectivity differences between individuals with AN and HC within thalamo-frontal circuits. An important limitation that future studies could address is the specificity of our findings by performing the same imaging analyses in other subcortical regions. In addition, it would be of interest to determine whether the frontal regions identified as having differential connectivity with the thalamus in this study also show abnormalities in morphology or functional connectivity with other brain regions, including the thalamus. A limitation inherent in resting-state functional MRI is the correlational nature of the measurement. To assess for differences in thalamo-frontal structural connectivity between individuals with AN and HC, additional imaging techniques such as diffusion tensor imaging with fiber tracking are needed. The cognitive measures in this study were selected because of their known relationship to the thalamo-frontal circuits under study; however, they have limitations. Although we found that working memory, as measured by the LNS, was significantly impaired in patients with AN in our study, other studies have not found impairment in this cognitive domain (Lao-Kaim et al, 2014; Nikendei et al, 2011). Cognitive flexibility is often impaired in AN (Steinglass et al, 2006; Wu et al, 2014), but 
findings with the Stroop and TMT have been inconsistent, perhaps because these tasks are less cognitively demanding. Furthermore, the relationship between these cognitive disturbances and the core behavioral disturbances in AN has not been established. Finally, although we did not detect effects of AN subtype on our outcome measures, it should be noted that these subtype analyses were underpowered due to small sample sizes comprising either subtype.

In conclusion, the current study begins to investigate thalamic dysfunction and the relationship with cognitive functioning in AN. Implicating thalamic dysfunction in AN adds to the growing literature suggesting CSTC loop dysfunction as an endophenotype of several disease states, including schizophrenia, ADHD, obsessive-compulsive disorder, and Tourette syndrome (Debes et al, 2014; Mitelman et al, 2005; Nigg and Casey, 2005; Posner et al, 2014a,b). Since alterations in thalamo-frontal connectivity might indicate general CSTC loop dysfunction, future studies could investigate whether other components of CTSC circuitry show alterations in AN. In addition, animal research may be a useful approach to test the causal relationship between altered functional connectivity and cognitive deficits with manipulations of thalamo-frontal interactions (eg, optogenetics) during behavioral performance of tasks probing these cognitive domains. Unveiling how CSTC loops contribute to cognitive function at a molecular, cellular, and system level using translational approaches may ultimately allow the generation of therapeutic interventions beneficial for both $\mathrm{AN}$ and other psychiatric conditions.

\section{FUNDING AND DISCLOSURE}

Dr Posner is a principal investigator on an investigatorinitiated grant from Shire Pharmaceuticals.

\section{ACKNOWLEDGMENTS}

This study was supported by NIMH grants R21-MH099388 (JS and JP), K23 MH76195 (JS), K23-MH091249 (JP), the Klarman Family Foundation (JS), and the Paul Janssen Fellowship in Translational Neuroscience Research (DB).

\section{REFERENCES}

Alexander GE, Crutcher MD (1990). Functional architecture of basal ganglia circuits: neural substrates of parallel processing. Trends Neurosci 13: 266-271.

Amianto F, Caroppo P, D'Agata F, Spalatro A, Lavagnino L, Caglio $\mathrm{M}$ et al (2013). Brain volumetric abnormalities in patients with anorexia and bulimia nervosa: a voxel-based morphometry study. Psychiatry Res 213: 210-216.

APA (2013). Diagnostic and Statistical Manual of Mental Disorders, 5th edn. American Psychiatric Association: Washington, DC, USA.

Bodell LP, Keel PK, Brumm MC, Akubuiro A, Caballero J, Tranel D et al (2014). Longitudinal examination of decision-making performance in anorexia nervosa: before and after weight restoration. J Psychiatr Res 56: 150-157.

Botvinick MM, Braver TS, Barch DM, Carter CS, Cohen JD (2001). Conflict monitoring and cognitive control. Psychol Rev 108: 624-652.

Bowie CR, Harvey PD (2006). Administration and interpretation of the Trail Making Test. Nat Protoc 1: 2277-2281.
Cazorla M, Shegda M, Ramesh B, Harrison NL, Kellendonk C (2012). Striatal D2 receptors regulate dendritic morphology of medium spiny neurons via Kir2 channels. J Neurosci 32: 2398-2409.

Cowdrey FA, Filippini N, Park RJ, Smith SM, McCabe C (2014). Increased resting state functional connectivity in the default mode network in recovered anorexia nervosa. Hum Brain Mapp 35: 483-491.

Debes N, Jeppesen S, Raghava JM, Groth C, Rostrup E, Skov L (2014). Longitudinal magnetic resonance imaging (MRI) analysis of the developmental changes of tourette syndrome reveal reduced diffusion in the cortico-striato-thalamo-cortical pathways. J Child Neurol 30: 1315-1326.

Decker JH, Figner B, Steinglass JE (2014). On weight and waiting: delay discounting in anorexia nervosa pretreatment and posttreatment. Biol Psychiatry 78: 606-614.

Delvenne V, Goldman S, De Maertelaer V, Simon Y, Luxen A, Lotstra F (1996). Brain hypometabolism of glucose in anorexia nervosa: normalization after weight gain. Biol Psychiatry 40: 761-768.

Delvenne V, Lotstra F, Goldman S, Biver F, De Maertelaer V, Appelboom-Fondu J et al (1995). Brain hypometabolism of glucose in anorexia nervosa: a PET scan study. Biol Psychiatry 37: $161-169$.

Ferguson BR, Gao WJ (2014). Development of thalamocortical connections between the mediodorsal thalamus and the prefrontal cortex and its implication in cognition. Front Hum Neurosci 8: 1027.

Forsyth JK, McEwen SC, Gee DG, Bearden CE, Addington J, Goodyear B et al (2014). Reliability of functional magnetic resonance imaging activation during working memory in a multisite study: analysis from the North American Prodrome Longitudinal Study. NeuroImage 97: 41-52.

Frank GK, Bailer UF, Meltzer CC, Price JC, Mathis CA, Wagner A et al (2007). Regional cerebral blood flow after recovery from anorexia or bulimia nervosa. Int J Eat Disord 40: 488-492.

Frieling $\mathrm{H}$, Fischer J, Wilhelm J, Engelhorn T, Bleich S, Hillemacher $\mathrm{T}$ et al (2012). Microstructural abnormalities of the posterior thalamic radiation and the mediodorsal thalamic nuclei in females with anorexia nervosa-a voxel based diffusion tensor imaging (DTI) study. J Psychiatr Res 46: 1237-1242.

Giguere M, Goldman-Rakic PS (1988). Mediodorsal nucleus: areal, laminar, and tangential distribution of afferents and efferents in the frontal lobe of rhesus monkeys. J Comp Neurol 277: 195-213.

Godart NT, Perdereau F, Rein Z, Berthoz S, Wallier J, Jeammet P et al (2007). Comorbidity studies of eating disorders and mood disorders. Critical review of the literature. J Affect Disord 97: 37-49.

Golden C (1978). Stroop Color and Word Test: A Manual for Clinical and Experimental Uses. Stoelting: Wood Dale, IL, USA.

Graybiel AM (2005). The basal ganglia: learning new tricks and loving it. Curr Opin Neurobiol 15: 638-644.

Haber SN, Calzavara R (2009). The cortico-basal ganglia integrative network: the role of the thalamus. Brain Res Bull 78: 69-74.

Hendrick OM, Ide JS, Luo X, Li CS (2010). Dissociable processes of cognitive control during error and non-error conflicts: a study of the stop signal task. PLoS One 5: e13155.

Hughes EJ, Bond J, Svrckova P, Makropoulos A, Ball G, Sharp DJ et al (2012). Regional changes in thalamic shape and volume with increasing age. NeuroImage 63: 1134-1142.

Husain MM, Black KJ, Doraiswamy PM, Shah SA, Rockwell WJ, Ellinwood EH Jr et al (1992). Subcortical brain anatomy in anorexia and bulimia. Biol Psychiatry 31: 735-738.

Jenkinson M, Bannister P, Brady M, Smith S (2002). Improved optimization for the robust and accurate linear registration and motion correction of brain images. NeuroImage 17: 825-841.

Jenkinson M, Beckmann CF, Behrens TE, Woolrich MW, Smith SM (2012). Fsl. NeuroImage 62: 782-790. 
Jenkinson M, Smith S (2001). A global optimisation method for robust affine registration of brain images. Med Image Anal 5: 143-156.

Johansen-Berg H, Behrens TE, Sillery E, Ciccarelli O, Thompson AJ, Smith SM et al (2005). Functional-anatomical validation and individual variation of diffusion tractography-based segmentation of the human thalamus. Cereb Cortex 15: 31-39.

Lao-Kaim NP, Giampietro VP, Williams SC, Simmons A, Tchanturia K (2014). Functional MRI investigation of verbal working memory in adults with anorexia nervosa. Eur Psychiatry 29: 211-218.

MacDonald AW 3rd, Cohen JD, Stenger VA, Carter CS (2000). Dissociating the role of the dorsolateral prefrontal and anterior cingulate cortex in cognitive control. Science 288: $1835-1838$.

Marenco S, Stein JL, Savostyanova AA, Sambataro F, Tan HY, Goldman AL et al (2012). Investigation of anatomical thalamocortical connectivity and FMRI activation in schizophrenia. Neuropsychopharmacology 37: 499-507.

Matsumoto R, Kitabayashi Y, Narumoto J, Wada Y, Okamoto A, Ushijima Y et al (2006). Regional cerebral blood flow changes associated with interoceptive awareness in the recovery process of anorexia nervosa. Prog Neuropsychopharmacol Biol Psychiatry 30: $1265-1270$

Milham MP, Banich MT, Claus ED, Cohen NJ (2003). Practicerelated effects demonstrate complementary roles of anterior cingulate and prefrontal cortices in attentional control. NeuroImage 18: 483-493.

Mitelman SA, Byne W, Kemether EM, Hazlett EA, Buchsbaum MS (2005). Metabolic disconnection between the mediodorsal nucleus of the thalamus and cortical Brodmann's areas of the left hemisphere in schizophrenia. Am J Psychiatry 162: 1733-1735.

Mitelman SA, Byne W, Kemether EM, Newmark RE, Hazlett EA, Haznedar MM et al (2006). Metabolic thalamocortical correlations during a verbal learning task and their comparison with correlations among regional volumes. Brain Res 1114: 125-137.

Moore AB, Li Z, Tyner CE, Hu X, Crosson B (2013). Bilateral basal ganglia activity in verbal working memory. Brain Lang 125: 316-323.

Niendam TA, Laird AR, Ray KL, Dean YM, Glahn DC, Carter CS (2012). Meta-analytic evidence for a superordinate cognitive control network subserving diverse executive functions. Cogn Affect Behav Neurosci 12: 241-268.

Nigg JT, Casey BJ (2005). An integrative theory of attention-deficit/ hyperactivity disorder based on the cognitive and affective neurosciences. Dev Psychopathol 17: 785-806.

Nikendei C, Funiok C, Pfuller U, Zastrow A, Aschenbrenner S, Weisbrod $\mathrm{M}$ et al (2011). Memory performance in acute and weight-restored anorexia nervosa patients. Psychol Med 41: 829-838.

Nugent AC, Martinez A, D'Alfonso A, Zarate CA, Theodore WH (2015). The relationship between glucose metabolism, restingstate fMRI BOLD signal, and GABAA-binding potential: a preliminary study in healthy subjects and those with temporal lobe epilepsy. J Cereb Blood Flow Metab 35: 583-591.
Park RJ, Godier LR, Cowdrey FA (2014). Hungry for reward: how can neuroscience inform the development of treatment for anorexia nervosa? Behav Res Ther 62: 47-59.

Parnaudeau S, O'Neill PK, Bolkan SS, Ward RD, Abbas AI, Roth BL et al (2013). Inhibition of mediodorsal thalamus disrupts thalamofrontal connectivity and cognition. Neuron 77: 1151-1162.

Patenaude B, Smith SM, Kennedy DN, Jenkinson M (2011). A Bayesian model of shape and appearance for subcortical brain segmentation. NeuroImage 56: 907-922.

Posner J, Marsh R, Maia TV, Peterson BS, Gruber A, Simpson HB (2014a). Reduced functional connectivity within the limbic cortico-striato-thalamo-cortical loop in unmedicated adults with obsessive-compulsive disorder. Hum Brain Mapp 35: 2852-2860.

Posner J, Park C, Wang Z (2014b). Connecting the dots: a review of resting connectivity MRI studies in attention-deficit/hyperactivity disorder. Neuropsychol Rev 24: 3-15.

Sowell ER, Thompson PM, Toga AW (2004). Mapping changes in the human cortex throughout the span of life. Neuroscientist 10: 372-392.

Steinglass JE, Walsh BT, Stern Y (2006). Set shifting deficit in anorexia nervosa. J Int Neuropsychol Soc 12: 431-435.

Swanson SA, Crow SJ, Le Grange D, Swendsen J, Merikangas KR (2011). Prevalence and correlates of eating disorders in adolescents. Results from the national comorbidity survey replication adolescent supplement. Arch Gen Psychiatry 68: 714-723.

Takano A, Shiga T, Kitagawa N, Koyama T, Katoh C, Tsukamoto E et al (2001). Abnormal neuronal network in anorexia nervosa studied with I-123-IMP SPECT. Psychiatry Res 107: 45-50.

Talbot A, Hay P, Buckett G, Touyz S (2014). Cognitive deficits as an endophenotype for anorexia nervosa: an accepted fact or a need for re-examination? The Int J Eat Disord 48: 15-25.

Tomasi D, Wang GJ, Volkow ND (2013). Energetic cost of brain functional connectivity. Proc Natl Acad Sci USA 110: 13642-13647.

Van Snellenberg JX, Slifstein M, Read C, Weber J, Thompson JL, Wager TD et al (2014). Dynamic shifts in brain network activation during supracapacity working memory task performance. Hum Brain Mapp 36: 1245-1264.

Wagner G, Koch K, Schachtzabel C, Schultz CC, Gaser C, Reichenbach JR et al (2013). Structural basis of the frontothalamic dysconnectivity in schizophrenia: a combined DCMVBM study. NeuroImage Clin 3: 95-105.

Wechsler D (1997). WAIS-III Administration and Scoring Manual. The Psychological Corporation: San Antonio, TX, USA.

Whitfield-Gabrieli S, Nieto-Castanon A (2012). Conn: a functional connectivity toolbox for correlated and anticorrelated brain networks. Brain Connect 2: 125-141.

Wu M, Brockmeyer T, Hartmann M, Skunde M, Herzog W, Friederich HC (2014). Set-shifting ability across the spectrum of eating disorders and in overweight and obesity: a systematic review and meta-analysis. Psychol Med 44: 3365-3385.

Yau WY, Bischoff-Grethe A, Theilmann RJ, Torres L, Wagner A, Kaye WH et al (2013). Alterations in white matter microstructure in women recovered from anorexia nervosa. Int J Eat Disord 46: 701-708.

Supplementary Information accompanies the paper on the Neuropsychopharmacology website (http://www.nature.com/npp) 